Discrete Comput Geom 28:29-44 (2002)

DOI: $10.1007 / \mathrm{s} 00454-001-0085-0$

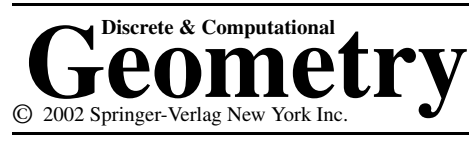

\title{
Examples and Counterexamples for the Perles Conjecture*
}

\author{
Christian Haase $^{1}$ and Günter M. Ziegler ${ }^{2}$ \\ ${ }^{1}$ Department of Mathematics, Duke University, \\ Durham, NC 27708-0320, USA \\ haase@math.duke.edu \\ ${ }^{2}$ MA 6-2, Institute of Mathematics, Technische Universität Berlin, \\ D-10623 Berlin, Germany \\ ziegler@math.tu-berlin.de
}

\begin{abstract}
The combinatorial structure of a $d$-dimensional simple convex polytopeas given, for example, by the set of the $(d-1)$-regular subgraphs of the facets-can be reconstructed from its abstract graph. However, no polynomial/efficient algorithm is known for this task, although a polynomially checkable certificate for the correct reconstruction exists.

A much stronger certificate would be given by the following characterization of the facet subgraphs, conjectured by Micha Perles: "The facet subgraphs of a simple d-polytope are exactly all the $(d-1)$-regular, connected, induced, non-separating subgraphs."

We present non-trivial classes of examples for the validity of the Perles conjecture: in particular, it holds for the duals of cyclic polytopes, and for the duals of stacked polytopes.

On the other hand, we observe that for any 4-dimensional counterexample, the boundary of the (simplicial) dual polytope $P^{\Delta}$ contains a 2-complex without a free edge, and without 2-dimensional homology. Examples of such complexes are known; we use a modification of "Bing's house" (two walls removed) to construct explicit 4-dimensional counterexamples to the Perles conjecture.
\end{abstract}

\section{Introduction}

If $P$ is a $d$-dimensional simple polytope, then its graph $G=G(P)$ is a $d$-regular, $d$ connected graph. If $F$ is any facet of $P$, then $G_{F}=G(F)$ is a $(d-1)$-regular, induced, connected, non-separating subgraph of $P$. Whether these properties already characterize

* The second author was supported by Deutsche Forschungsgemeinschaft (DFG). 
the subgraphs of facets of a simple polytope was asked by Perles [19] a long time ago:

Does every $(d-1)$-regular, induced, connected, non-separating subgraph of the graph of a simple d-polytope correspond to a facet of $P$ ?

For $d \leq 2$ this is trivially true, for $d=3$ it is equivalent to a basic result of Whitney [22].

Perles' question is important in the context of the reconstruction of polytopes. (See [15] for a general source on reconstruction of polytopes, and in particular for Perles' unpublished work in this field.) Simple polytopes can theoretically be reconstructed from their graphs - this was originally conjectured by Perles, and first proved by Blind and Mani [4], and particularly elegantly by Kalai [14]. (See also [12].) However, although Kalai's proof can be implemented to reconstruct polytopes of reasonable size [1], the reconstruction is far from easy or efficient (in the theoretical or practical sense).

Using ideas from Kalai's paper [14], Joswig et al. [13] derived a certificate for the reconstruction of a simple polytope that can indeed be checked in polynomial time. They do, however, not (yet) have a polynomial time algorithm to find/construct such a certificate. If Perles' question had a positive answer, then the reconstruction would be much easier, as was noted by Kalai [14], and by Achatz and Kleinschmidt [1]. In particular, the Perles conjecture proposes an efficient criterion for recognizing the facet subgraphs of simple polytopes, and thus an easy check whether a given list of subgraphs is indeed the complete list of facet subgraphs.

Here we first establish that the Perles conjecture is true for non-trivial classes of polytopes, including all the duals of stacked polytopes, and the duals of cyclic polytopes (Section 3).

However, our main result is that, perhaps surprisingly, the Perles conjecture is not true in general. To prove this, we first identify certain topological obstructions for the Perles conjecture (Section 4). We then take (known) 2-dimensional complexes that realize this obstruction - the simplest one to handle being known as "Bing's house with two rooms" (with two walls removed). We describe fairly general methods to produce counterexamples from such 2-complexes.

Using the polymake framework [7], [8], Nikolaus Witte [23] has produced a "small" explicit counterexample, a simple 4-polytope with $f_{0}=2592$ vertices and $f_{3}=529$ facets, along the prescriptions of this paper. It will be presented as an electronic geometry model at www.eg-models.de.

\section{Versions of the Perles Conjecture}

We distinguish two slightly different versions of the Perles conjecture, where the first and stronger version is the one stated in [14], see also Problem 3.13* of [25].

Definition 1. A simple $d$-polytope $P$ satisfies the Perles conjecture if every induced, connected, $(d-1)$-regular, non-separating subgraph of $G(P)$ is the graph of a facet of $P$.

A simple $d$-polytope $P$ satisfies the weak Perles conjecture if every induced, $(d-1)$ connected, $(d-1)$-regular, non-separating subgraph of $G(P)$ is the graph of a facet of $P$. 
If $P$ is a simple polytope, then its polar dual $P^{\Delta}$ is simplicial. The vertices and edges of $P$ correspond to the facets and ridges of $P^{\Delta}$. The different notions in the Perles conjecture for $P$ have immediate translations to the combinatorics of $P^{\Delta}$. This yields the "puzzle" reformulation of Blind and Mani [4].

- An induced subgraph $H$ of the graph of $P$ corresponds to a collection of facets and ridges of $P^{\Delta}$ such that whenever two adjacent facets belong to the collection, then their common ridge is also in the collection. We will present this collection of facets and ridges in terms of the pure simplicial $(d-1)$-complex $\Gamma(H)$ that it generates. The dual graph of this "pseudomanifold" is the one that the Perles conjecture in the original form refers to.

- The induced subgraph $H$ is $(d-1)$-regular if and only if every $(d-1)$-simplex in $\Gamma(H)$ has exactly one free $(d-2)$-simplex. (A simplex is free in a simplicial complex if it is contained in exactly one other/larger face of the complex.)

- The induced subgraph $H$ is connected if and only if the corresponding subcomplex $\Gamma(H)$ is dually connected, that is, any two $(d-1)$-simplices can be joined by a chain of successively adjacent facets and ridges. (This is often called "strongly connected" for pseudomanifolds.)

- The induced subgraph does not separate its complement (graph theoretically) if and only if the corresponding subcomplex does not separate its complement (topologically): this is true since the topological complement retracts to the "dual block complex" [17] whose 1-skeleton is the graph of the complement.

- If $H$ is the graph of a facet, then the corresponding subcomplex $\Gamma(H)$ is a vertex star: the collection of all facets that contain a given vertex.

Definition 2. A simplicial $d$-polytope satisfies the Perles conjecture if the vertex stars are the only pure $(d-1)$-dimensional, dually connected, non-separating subcomplexes of its boundary for which every maximal simplex has exactly one free face.

A simplicial $d$-polytope satisfies the weak Perles conjecture if the vertex stars are the only pure $(d-1)$-dimensional, dually $(d-1)$-connected, non-separating subcomplexes of its boundary for which every maximal simplex has exactly one free face.

\section{Positive Results}

Before embarking on the construction of counterexamples, and as an indication of why these need to be rather complicated, we demonstrate here that the Perles conjecture is not only quite plausible, but that it is even true for large classes of examples: it is certainly not an "unreasonable" conjecture. (See also [5] for positive evidence toward the Perles conjecture.)

We may safely assume that Perles himself verified his conjecture for non-trivial classes of examples; the Diplomarbeit of Stolletz [21] also has a number of positive results. Thus, the Perles conjecture is true, for example, for all $d$-dimensional polytopes with $d \leq 3$ (even without a restriction to simple/simplicial polytopes), for all 4-dimensional simple polytopes with at most eight vertices [21, Section 5.2.1], for all 4-dimensional product 
polytopes [21, Kapitel 2], for all 4-dimensional cyclic polytopes [21, Kapitel 4], and for all stacked polytopes [21, Satz 3.1.3].

Indeed, the treatment of vertex truncations, and thus of stacked polytopes, presents no greater difficulties.

Proposition 1 [21, Lemma 3.1.2]. Truncation( "cutting off a simple vertex") preserves the validity of the Perles conjecture: a vertex-truncated simple d-polytope $P^{\prime}:=\tau_{v}(P)$ satisfies the Perles conjecture if and only if P satisfies it.

Corollary 2 [21, Satz 3.1.3]. All stacked polytopes (dually: the multiple vertex-truncations of simplices) satisfy the Perles conjecture.

We now develop a rather systematic method to prove the Perles conjecture for classes of simple polytopes. We demonstrate its use for products, for wedges, and in particular for the duals of cyclic polytopes. Here is the idea: Let $P$ be a simple $d$-polytope, $G=G(P)$ its graph, with vertex set $V$. We are interested in $(d-1)$-regular induced connected subgraphs $H$ that do not separate; as induced subgraphs they are given by their vertex sets $V_{H} \subset V$. The "Ansatz" is to concentrate on the vertex set $\bar{V}=V \backslash V_{H}$ of the complement of $H$ in $G$. In the first step we (try to) classify all those vertex sets $\bar{V} \subset V$ for which

(1) the induced subgraph $G[\bar{V}]$ is connected, and

(2) $\bar{V}$ is triangle-closed and quadrilateral-closed: if $\bar{V}$ contains "all but one" vertices of a triangle or quadrilateral of $G$, then it must contain all its vertices.

The first condition is necessary since we want $H$ to be non-separating. If the second condition is violated, then the "missing vertex" has two neighbors in $\bar{V}$, and thus has degree at most $d-2$ in $H$.

In the second step we then identify those sets $\bar{V}$ for which every vertex not in $\bar{V}$ has exactly one neighbor in $\bar{V}$. Our hope is then to end up with only the (complements of) facet subgraphs, plus the trivial case given by $\bar{V}=V$.

Proposition 3. If two simple polytopes $P_{1}$ and $P_{2}$ satisfy the Perles conjecture, then so does their product $P_{1} \times P_{2}$.

Proof. Let $H \subset G\left(P_{1} \times P_{2}\right)=G_{1} \times G_{2}$ be a $\left(d_{1}+d_{2}-1\right)$-regular subgraph that is connected and does not separate $\left(d=d_{1}+d_{2}\right)$. Now (Step 1) if the complement vertex set $\bar{V}$ satisfies (1) and (2), then it is a product set, $\bar{V}=\bar{V}_{1} \times \bar{V}_{2}$.

Now (Step 2) if we can choose $v_{i} \in V_{i} \backslash \bar{V}_{i}$ for $i=1,2$, then this yields a vertex $\left(v_{1}, v_{2}\right) \in H$ that in $G_{1} \times G_{2}$ has graph-theoretic distance at least 2 from the complement set $\bar{V}_{1} \times \bar{V}_{2}$ (symbolized by the vertex $\bullet$ in Fig. 1). Thus this vertex of $H$ has degree $d_{1}+d_{2}$, which is impossible.

Hence we get that the $\left(d_{1}+d_{2}-1\right)$-regular, induced, connected and non-separating subgraphs all have the form $H=G_{1} \times H_{2}$ or $H=H_{1} \times G_{2}$, where $H_{i} \subset G_{i}$ is $\left(d_{i}-1\right)$ regular, induced, connected and non-separating. By the Perles conjecture, which is valid for $P_{i}$, it follows that $H_{i}$ is the graph of a facet of $P_{i}$, and hence that $H$ is the graph of a facet of $P_{1} \times P_{2}$. 


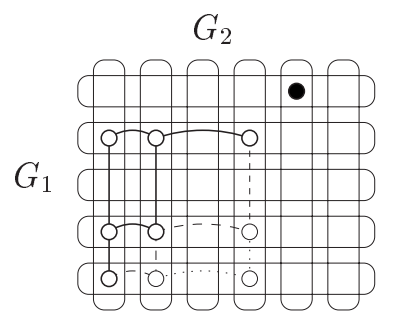

Fig. 1. Property (2) at work in a product graph.

We refer to Klee and Walkup [16] and to Holt and Klee [11] for the construction of the wedge $\omega_{F} P$ of a polytope $P$ over a facet $F$. (It is what you think it ought to be.)

Proposition 4. If $P$ is a simple d-polytope that satisfies the Perles conjecture, and $F$ is a facet of $P$, then $\omega_{F}(P)$ satisfies the Perles conjecture (and conversely).

Sketch of Proof. The vertex set of $P^{\prime}:=\omega_{F}(P)$ may be decomposed as $V_{F} \cup V_{T} \cup V_{B}$, into the vertices that lie in the face $F$ resp. not in the bottom facet resp. not in the top facet of the wedge (Fig. 2). In Step 1 one then verifies that if $\bar{V}$ meets both $V_{B}$ and $V_{T}$, then it always contains "none or both" from a pair of corresponding top and bottom vertices.

Lemma 5. If $d>2$ is odd, then $C_{d}(n)^{\Delta}$ is combinatorially equivalent to $\omega_{F} C_{d-1}(n-1)^{\Delta}$, for a facet $F \cong C_{d-2}(n-2)^{\Delta}$. If $d>1$ is even, and $n=d+1$, then $C_{d}(n)^{\Delta}$ is a d-simplex; for $n=d+2$ it is a product of two $\frac{d}{2}$-simplices.

Proof. To be derived from Gale's evenness criterion; see [9] or [25].

Theorem 6. The cyclic polytopes $C_{d}(n)$ satisfy the Perles conjecture.

Proof. We work with the duals, $P=C_{d}(n)^{\Delta}$. By Propositions 3 and 4 plus Lemma 5, we need only treat the case where $d=2 e$ is even, and $n>d+2$.

(i) The graph $G=G\left(C_{d}(n)^{\Delta}\right)$ has a simple combinatorial description, via Gale's evenness criterion: Its vertices $v$ correspond to those subsets $S \in\left(\begin{array}{c}{[n]} \\ d\end{array}\right)$ which split into a union of $e$ adjacent pairs modulo $n$ (that is, we identify the elements of $[n]=\{1, \ldots, n\}$

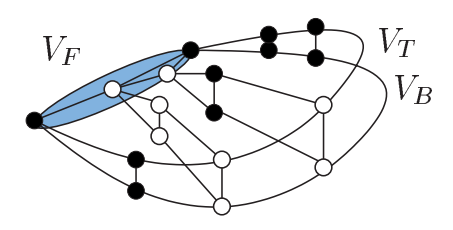

Fig. 2 


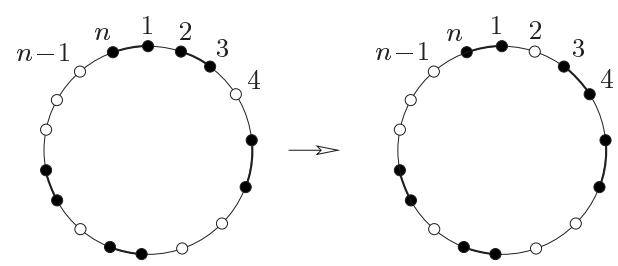

Fig. 3. One single pair is moved, thus we get an edge of $G$, and of $G^{\prime}$, corresponding to $12356 \ldots \rightarrow$ $13456 \ldots$

with $\mathbb{Z}_{n}$ ). The splitting into adjacent pairs is always unique. We call a "block" any nonempty union of adjacent pairs that is contiguous, that is, without a gap. Two vertices are adjacent if they differ in a single element, that is, if one arises from the other by moving one block by "one unit." This also provides a canonical orientation on each edge: we put a directed edge $v_{S} \rightarrow v_{T}$ if we get from $S$ to $T$ by moving a block "up" $(\bmod n)$. The resulting digraph is, of course, not acyclic. We also consider the subgraph $G^{\prime} \subseteq G$, which has the same vertex set as $G$, but only retains those directed edges that correspond to moves of single pairs (Fig. 3). (In Fig. 4, this is the graph formed by the straight edges only.) The structure of the digraph $G^{\prime}$ is closely linked to the poset

$$
L:=\left\{\left(j_{1}, j_{2}, \ldots, j_{d}\right) \in \mathbb{Z}^{d}: j_{2 k}=j_{2 k-1}+1, j_{2 k+1} \geq j_{2 k}+1, j_{d} \leq j_{1}+n-1\right\},
$$

equipped with componentwise partial order. This poset is a distributive lattice. Moreover, reduction modulo $n$ defines a surjective, and locally injective, digraph map $\Phi: L \longrightarrow G^{\prime}$, from the Hasse diagram of $L$ onto the digraph $G^{\prime}$.

(ii) Now assume (Step 1) that $\bar{V} \subset V$ satisfies (1) and (2). Every move of a block can be decomposed into a sequence of moves of pairs. Thus property (2) implies that $G^{\prime}[\bar{V}]$ is also connected (as an undirected graph): every directed arc in $G[\bar{V}]$ canonically corresponds to a sequence of directed arcs in $G^{\prime}[\bar{V}]$. Thus $G[\bar{V}]$ is acyclic if and only if $G^{\prime}[\bar{V}]$ is acyclic.

(iii) Next we treat the case that $G^{\prime}[\bar{V}]$ contains a directed cycle. Every such cycle lifts into a two-way infinite, maximal chain $C \subseteq \Phi^{-1}(\bar{V})$ in the lattice $L$. Every element $w \in L$ is contained in a finite interval $\left[v^{\prime}, v^{\prime \prime}\right]$ between elements $v^{\prime}, v^{\prime \prime} \in C$. This $C$ restricts to a maximal chain $\gamma_{0}$ in the interval $\left[v^{\prime}, v^{\prime \prime}\right]$, while $w$ will lie on some other maximal chain $\gamma$ of this interval. In a distributive (and hence semimodular) lattice one can move from any maximal chain to any other one by one-element exchanges, see Björner [3, Section 6]. This implies via (2) not only the elements of $\gamma_{0}$, but also all elements of chains that we can move to, belong to $\Phi^{-1}(\bar{V})$. Thus we get $w \in \Phi^{-1}(\bar{V})=L$, and hence $\bar{V}=V$.

(iv) Thus we may assume that $G^{\prime}[\bar{V}]$ is acyclic. We can then identify $G[\bar{V}]$ with an induced subgraph of the Hasse diagram of $L$ : every connected component of $\Phi^{-1}(\bar{V})$ is isomorphic to $G^{\prime}[\bar{V}]$. With this, properties (1) and (2), and the lower and upper semimodularity of the lattice $L$, imply that $\bar{V}$ corresponds to an interval in $L$ (see again [3]); that is, there are elements $x_{0}, x_{1} \in L$ such that $G^{\prime}[\bar{V}]$ is isomorphic to the Hasse diagram of the interval $\left[x_{0}, x_{1}\right] \subseteq L$. This corresponds to unique vertices $v_{0}, v_{1} \in \bar{V}$ such that $\bar{V}$ consists of all vertices of $G^{\prime}$ that lie on a directed path of minimal length from $v_{0}$ to $v_{1}$.

(v) Assume that $\bar{V}$ contains no "no-gap vertex," whose set $S$ consists of one single 

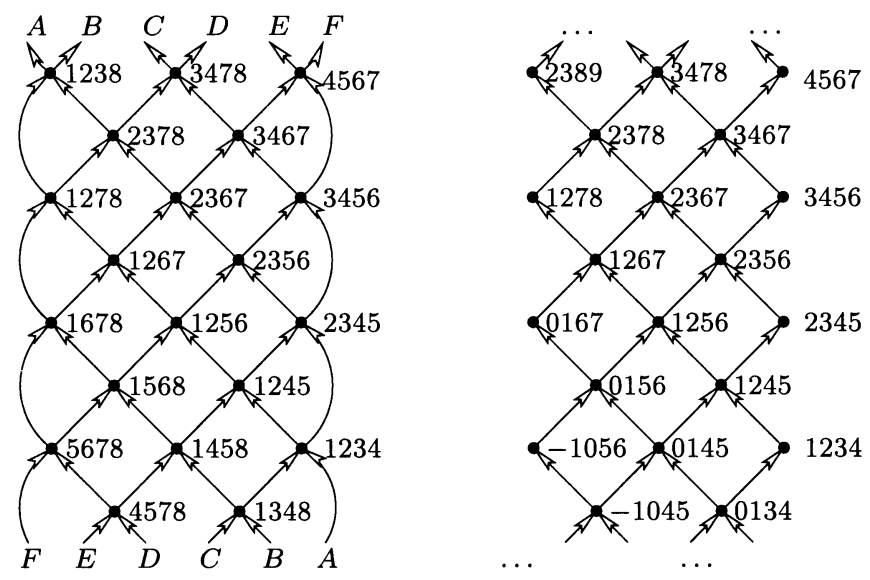

Fig. 4. This depicts the digraph $G$, and the lattice $L$, for $d=4, n=8$. The digraph $G$ is finite, the ends are to be identified according to the capital letters. It is not planar, but for $d=4$ it embeds into a Möbius band. The lattice $L$ is infinite.

block of size $d$. (In the case $d=4$, see Fig. 4, this means that $\bar{V}$ contains no vertex on the border of the Möbius strip.) Then every no-gap vertex needs to have exactly one neighbor in $\bar{V}$, which is a one-gap vertex consisting of $d$ elements from a block of $d+1$ adjacent vertices. Every such block corresponds to a $\left(\frac{d}{2}+1\right)$-clique; these cliques contain two no-gap vertices each, and each no-gap vertex is contained in two of these $\left(\frac{d}{2}+1\right)$-cliques. (In Fig. 4 this corresponds to the chain of triangles in the boundary of the Möbius strip.)

Now we lift the situation to the lattice $L$, where the chain of cliques gives rise to $d / 2$ distinct chains, which are disjoint by our assumption $n>d+2$. The interval $\left[x_{0}, x_{1}\right]$ can contain at most one element from each of the chains. Thus it can contain at most $d / 2$ elements from the chains. Thus at most $d$ no-gap vertices are adjacent to a vertex in $\left[x_{0}, x_{1}\right]$. Projecting this back to $G$, we find that at most $d$ no-gap vertices are adjacent to a vertex in $\bar{V}$. But they all have to be, so we get $n \leq d$, a contradiction.

(vi) If $\bar{V}$ contains a one-block vertex, then we see, using (2), that both $v_{0}$ and $v_{1}$ must be one-block vertices. By symmetry, we may then assume that $S_{0}=\{1,2, \ldots, d\}$ and $S_{1}=\{k+1, \ldots, d+k-1\}$ for some $k$. This ends Step 1, and Step 2-the identification of those parameters $k$ for which each vertex in $V \backslash \bar{V}$ has exactly one neighbor in $\bar{V}$-is now easy.

\section{The Obstruction}

We now restrict our discussion to the case $d=4$, where we will construct a counterexample to the weak Perles conjecture. ${ }^{1}$ From it, one gets counterexamples to the weak Perles conjecture in all dimensions $d>4$ from the constructions of Section 3, such as wedges and products.

\footnotetext{
${ }^{1}$ The figures try to illustrate the situation in $d=3$, though.
} 
The discussion in this section motivates our construction; it leads us to well-characterized obstructions to the validity of the Perles conjecture (for any specific polytope). For simplicity, we formulate this for $d=4$; the generalization to $d \geq 4$ is immediate.

Proposition 7. Let $P^{\Delta}$ be a simplicial 4-polytope whose boundary complex $\Delta:=$ $\Delta\left(\partial P^{\Delta}\right)$ contains a dually connected pure 3-dimensional subcomplex $\Gamma$, all whose tetrahedra have exactly one free triangle in $\Gamma$. Then $\Gamma$ (and in particular $\Delta$ ) contains a pure 2-dimensional, dually connected subcomplex core $(\Gamma)$ without free edges. Moreover, $\Gamma$ separates the boundary of $P^{\Delta}$ if $\operatorname{core}(\Gamma)$ does, and core $(\Gamma)$ is empty if and only if $\Gamma$ is a vertex star.

In the case $d=3$, core $(\Gamma)$ would be 1 -dimensional, with neither a cycle (nonseparating) nor a free face: a leafless tree. Thus core $(\Gamma)$ would necessarily be empty, and $\Gamma$ is a vertex star. This (re)proves Whitney's theorem (Perles' conjecture for 3-polytopes).

Proof. We specify a pure 2-dimensional subcomplex core $(\Gamma)$ of $\Gamma$ which will comply with our conditions (the definition is due to Carsten Schultz): Every tetrahedron $\sigma \in \Gamma$ has a unique vertex $v(\sigma)$ opposite to its free face. A triangle $\tau$ belongs to core $(\Gamma)$ if both its neighboring tetrahedra $\sigma_{1}, \sigma_{2}$ belong to $\Gamma$, and if $v\left(\sigma_{1}\right) \neq v\left(\sigma_{2}\right)$.

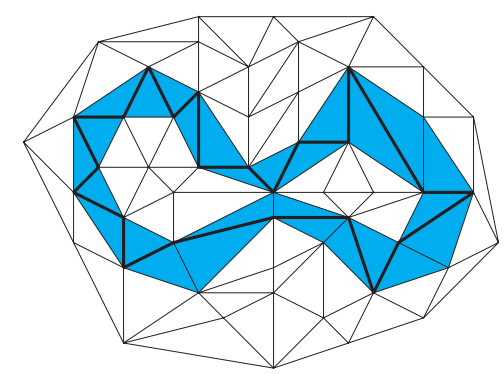

Fig. 5. If the grey 2-complex is $\Gamma$, then the black 1-dimensional subcomplex is its core.

Claim 1. The complex core $(\Gamma)$ has no free edge.

Suppose that $\varrho$ is a free edge of the triangle $\tau \in \operatorname{core}(\Gamma)$. The tetrahedra of $\Delta$ which contain $\varrho$ are cyclically ordered: $\sigma_{1}, \ldots, \sigma_{n}$, as in Fig. 6 . We can assume that $\tau=\sigma_{1} \cap \sigma_{2}$ and $\sigma_{3} \in \Gamma$. For, if $\sigma_{3}$ and $\sigma_{n}$ were both not in $\Gamma$, then this would imply $v\left(\sigma_{1}\right)=v\left(\sigma_{2}\right)$, and hence $\tau \notin \operatorname{core}(\Gamma)$.

Because $\sigma_{2} \cap \sigma_{3} \notin \operatorname{core}(\Gamma)$, we have $v\left(\sigma_{3}\right)=v\left(\sigma_{2}\right)$. This vertex lies in $\sigma_{2} \cap \sigma_{3}$, and it cannot be the vertex of $\sigma_{2}$ that is opposite to $\tau$; thus we get that $v\left(\sigma_{3}\right)=v\left(\sigma_{2}\right) \in \varrho$. In particular, this means that $\sigma_{3} \cap \sigma_{4}$ is not the free face of $\sigma_{3}$, and thus $\sigma_{4} \in \Gamma$. Iterating these arguments, we see that $\sigma_{1}, \ldots, \sigma_{n} \in \Gamma$ and $v\left(\sigma_{n}\right)=\cdots=v\left(\sigma_{2}\right) \neq v\left(\sigma_{1}\right)$. So, $\sigma_{n} \cap \sigma_{1}$ is another triangle in core $(\Gamma)$. 


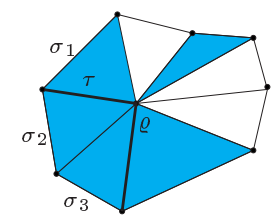

Fig. 6. Illustration of Claim 1.

Claim 2. The complex core $(\Gamma)$ is empty if and only if $\Gamma$ is a vertex star.

If core $(\Gamma)$ is empty, as $\Gamma$ is dually connected, all the vertices $v(\sigma)$ are equal, say, $v_{0}$. Hence $\Gamma$ is part of $\operatorname{star}_{\Delta}\left(v_{0}\right)$. But then $\Gamma$ must be the whole star, because there is only one free face per tetrahedron.

\section{Claim 3. If core $(\Gamma)$ separates, then so does $\Gamma$.}

Alexander duality implies that a subcomplex of a 3-sphere separates if and only if it has non-trivial 2-dimensional homology. The 3-dimensional complex $\Gamma$ collapses down to a 2-dimensional subcomplex $\Gamma^{\prime}$, which contains core $(\Gamma)$. Since $\Gamma^{\prime}$ has dimension 2 , it is clear that the (relative) homology group $\mathrm{H}_{3}\left(\Gamma^{\prime}\right.$, core $\left.(\Gamma)\right)$ vanishes. Hence, the map $\iota$ in the exact sequence

$$
\begin{aligned}
& \mathrm{H}_{3}(\Gamma, \operatorname{core}(\Gamma)) \longrightarrow \mathrm{H}_{2}(\operatorname{core}(\Gamma)) \stackrel{\iota}{\longrightarrow} \mathrm{H}_{2}(\Gamma) \longrightarrow \cdots \\
& \mathrm{H}_{3}\left(\Gamma^{\prime}, \operatorname{core}(\Gamma)\right)=0
\end{aligned}
$$

is injective, and the claim is proved.

We further analyze the above situation. The aim is to reveal the combinatorial properties of complexes that appear as a core (in addition to the topological ones that we have already seen). In Section 5 we show how to guarantee these properties using stellar subdivisions.

Consider now the structure of $\Gamma$ locally, restricted to the (open) star of a vertex $v_{0}$. This vertex star is divided by core $(\Gamma)$ into several pieces and $\Gamma$ is a union of some of these pieces:

Lemma 8. Let $\sigma, \sigma^{\prime} \in \operatorname{star}_{\Delta}\left(v_{0}\right)$ be tetrahedra in the same piece, that is, such that there is a dual path in $\operatorname{star}_{\Delta}\left(v_{0}\right)$ between $\sigma$ and $\sigma^{\prime}$ that does not meet $\operatorname{core}(\Gamma)$. If $\sigma \in \Gamma$ and $v(\sigma)=v_{0}$, then $\sigma^{\prime} \in \Gamma$ and $v\left(\sigma^{\prime}\right)=v_{0}$.

Corollary 9. For every vertex $v_{0}$ of core $(\Gamma)$ the complex which is generated by $\left\{\sigma \in \Gamma: v(\sigma)=v_{0}\right\}$ is the closure of a union of components of $\operatorname{star}_{\Delta}\left(v_{0}\right) \backslash \operatorname{core}(\Gamma)$. Furthermore, two such components that correspond to different $v_{0}$ 's intersect at most in codimension 1, and if so, then this intersection is included in core $(\Gamma)$. 


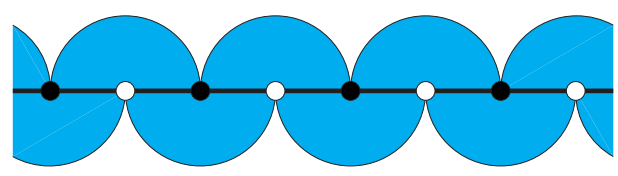

Fig. 7. Illustration of Corollary 9.

\section{A Counterexample}

Proposition 7 makes one ask for a 2-dimensional simplicial complex $\Gamma$, without a free edge (every edge is contained in at least two triangles), which does not separate (that is, $\mathrm{H}_{2}(\Gamma, \mathbb{Z})=\{0\}$ ), and which is embeddable in $\mathbb{R}^{3}$ (in particular, $\mathrm{H}_{1}(\Gamma, \mathbb{Z})$ has no torsion). Such 2-dimensional complexes do exist. The most prominent examples are probably Borsuk's "dunce hat" [6], [24], and Bing's "house with two rooms" [2], [10, p. 4], which are even contractible.

To keep the constructions for the following simpler, we put an extra condition on the complexes we look at: we want them to be "essentially manifolds," that is, we want every point to have a closed neighborhood that is homeomorphic either to two or to three triangles that are joined together at a common edge. (Thus, we admit singular curves, along which a manifold branches into three parts, but we do not admit singular points that may be more complicated than the points on a singular curve.)

In the following we provide an as-concrete-as-possible description of a specific counterexample of this type. Further counterexamples of the same type may be obtained along the same lines.

Our point of departure is a modification $\mathcal{B}$ of Bing's house. Two extra walls are missing that would usually be added to make the interior of each room simply connected (see Fig. 8).

This $\mathcal{B}$ can be embedded into a pile of $2 \times 3 \times 4$ cubes- a cubical complex. Triangulate the pile by the arrangement of hyperplanes $x_{i}-x_{j}=k$ to get a simplicial complex (see Fig. 9). Add a cone over the boundary to get the boundary of a simplicial 4-polytope $Q^{\Delta}$ (any subdivision by a hyperplane arrangement is "coherent" /"regular"; see [25,

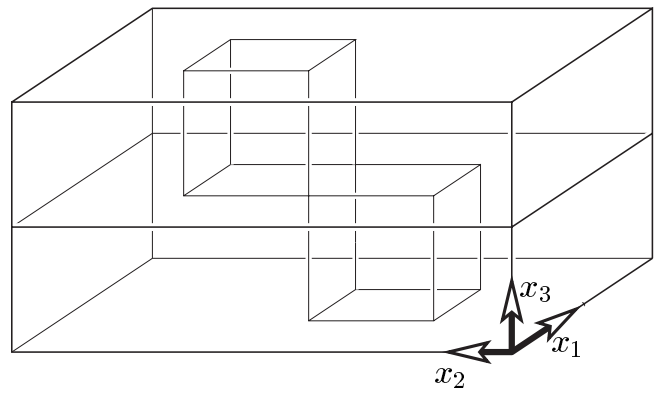

Fig. 8. The complex $\mathcal{B}$ has two rooms: the downstairs room is connected to the outside via the chimney through the upstairs room, the upstairs room is connected to the outside via the chimney through the downstairs room. 


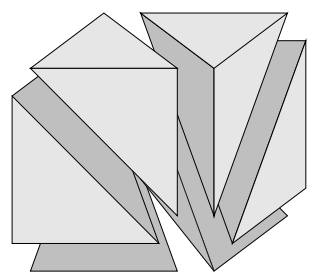

Fig. 9

Lecture 5]). This polytope contains a triangulation of $\mathcal{B}$ as a subcomplex, which we also denote by $\mathcal{B}$. The vertices of this subcomplex now get "colors" 0,1 , or 2 , by assigning to each vertex the sum of its coordinates modulo 3 . The only edges that join vertices of the same color are the diagonals of the cubes, which are not in $\mathcal{B}$. The two chimneys look as in Fig. 10.

The upper chimney:
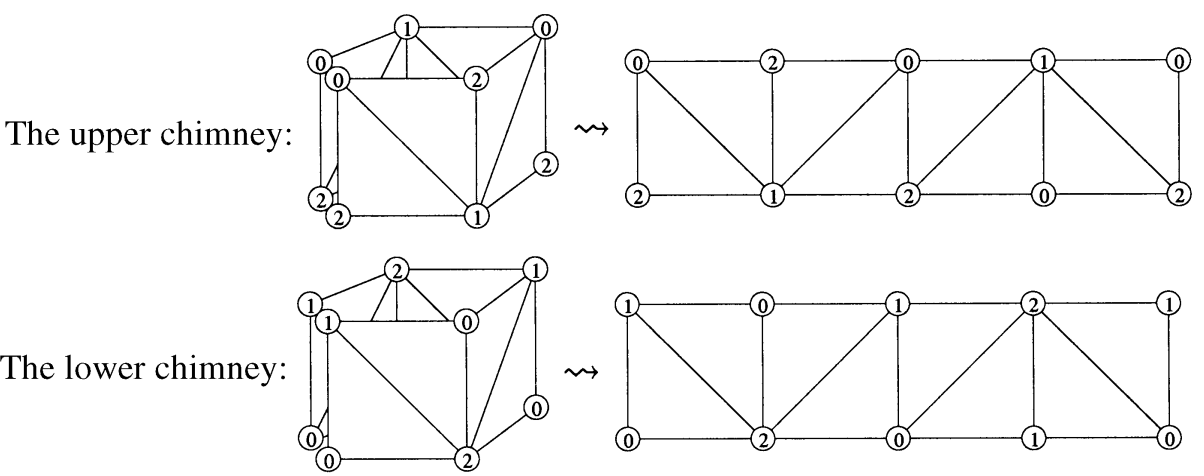

Fig. 10

We now perform a sequence of stellar subdivisions on faces of $Q^{\Delta}$, as follows:

- Perform stellar subdivisions on vertical edges in the chimneys, namely in the upper chimney on the two edges with labels ${ }_{2}^{0}$, and in the lower chimney on the two edges with labels ${ }_{0}^{1}$ (see Fig. 11).

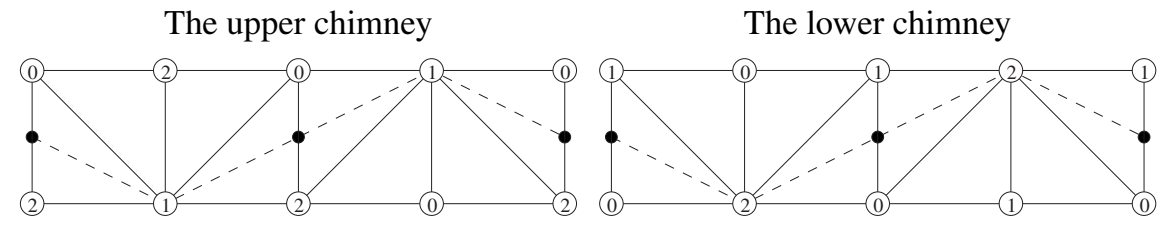

Fig. 11

The resulting new edges in the chimneys (four each; dashed edges in Fig. 11) are interpreted as marking the boundary between "inside" and "outside" in the chimneys. No color is given to these four new vertices. 
With the dashed separation edges in place, we have now achieved the situation that every triangle in the core is adjacent to exactly two of "outside," "upstairs" and "downstairs." Furthermore, if a triangle is adjacent to "outside," then it has a 0 -colored vertex, for "upstairs" it has a 1-colored vertex, and for "downstairs" a 2-colored vertex.

- Now perform stellar subdivisions on all simplices not in $\mathcal{B}$ for which all proper faces belong to $\mathcal{B}$ (all of these faces are edges). Thus $\mathcal{B}$ becomes an induced subcomplex.

For example, this operation needs to be performed on the main diagonals of the original cubes.

- Whenever there are two non-adjacent vertices $v_{1}, v_{2} \in \mathcal{B}$ and a vertex $w \notin \mathcal{B}$ such that both $\left\{w, v_{1}\right\}$ and $\left\{w, v_{2}\right\}$ are edges, perform a stellar subdivision on one of these edges. After these operations, we have achieved the property that for any two non-adjacent vertices of $\mathcal{B}$, the intersection of their stars is contained in $\mathcal{B}$ (it may be empty).

For example, such subdivisions will be made for $v_{1}, v_{2}$ taken to be the endpoints of a main cube diagonal and $w$ the new subdivision vertex on the diagonal, and when $v_{1}, v_{2}$ are on the boundary of the pile of cubes and $w$ the cone vertex.

(A more efficient construction, sufficient for our purpose, would perform the subdivisions only if $v_{1}$ and $v_{2}$ have the same color, or if they are both adjacent to the same vertex of $\mathcal{B}$ on the boundary between "inside" and "outside" in one of the chimneys.)

The result of all these operations is now called $P^{\Delta}$. Thus the polytope $P$, a counterexample to Perles' original conjecture, is obtained as its polar dual. It remains to name the vertex star pieces that we want to attach to $\mathcal{B}$ in order to get a Perles-contradicting subcomplex. For that purpose we use the vertex labels, and proceed according to the scheme

\begin{tabular}{lll}
\hline Label & & Partial vertex star \\
\hline 0 & $\longleftrightarrow$ & outside \\
1 & $\longleftrightarrow$ & upstairs room \\
2 & $\longleftrightarrow$ & downstairs room \\
\hline
\end{tabular}

If a vertex with label 2 does not touch the downstairs room, then no partial vertex star is assigned, etc. The union $\Gamma$ of these partial vertex stars is a non-separating subcomplex, whose tetrahedra all have exactly one free triangle opposite to "their" vertex of $\mathcal{B}$; this is guaranteed by the intersection property of the vertex stars discussed above.

\section{Connectivity}

Finally, we verify that our counterexample does not even satisfy the weak Perles conjecture, that is, $\Gamma$ is dually 3 -connected. Our arguments will be general enough to work as well for other counterexamples of the same type (where the core is essentially a manifold). Of course, for specific counterexamples, 3-connectedness could as well be verified by explicit computer calculation. This has been done for the example by N. Witte [23], to be presented in www.eg-models.de. 
Remove two tetrahedra $\bar{\sigma}_{1}, \bar{\sigma}_{2}$ from $\Gamma$. We want to join any two remaining tetrahedra $\sigma_{0}, \sigma_{\infty}$ by a dual path, avoiding $\bar{\sigma}_{1}, \bar{\sigma}_{2}$. This path is constructed in three steps:

1. Join $\sigma_{0}, \sigma_{\infty}$ to tetrahedra which have a full-dimensional intersection with core $(\Gamma)$.

2. Join these full-dimensional intersections by a dual path in $\operatorname{core}(\Gamma)$.

3. Lift this path to a path in $\Gamma$.

Step 1 follows from a simple fact about graphs of 3-polytopes:

Lemma 10. Every tetrahedron $\sigma \in \Gamma$ can be joined to some tetrahedron with fulldimensional intersection with core $(\Gamma)$, avoiding $\bar{\sigma}_{1}, \bar{\sigma}_{2}$.

Proof. The dual graph of the star of $v(\sigma)$ in $\Delta$ corresponds to a (simple) 3-dimensional polytope, and is therefore 3-connected. Any dual path from $\sigma$ to a tetrahedron in a different component has to pass through $\operatorname{core}(\Gamma)$.

For Step 2, we need some preparation. In view of Step 3, we consider a graph $G_{\mathcal{B}}^{*}$ defined as follows: its nodes are the 2-faces of $\operatorname{core}(\Gamma)$, and its edges correspond to pairs $\left(\tau_{1}, \tau_{2}\right)$ of 2 -faces which share a 1 -face $\varrho$, and which can be joined by a dual path in $\operatorname{star}(\varrho ; \Gamma) \backslash \operatorname{core}(\Gamma)$; see Fig. 12. We are interested in the subgraphs of $G_{\mathcal{B}}^{*}$ that are induced by those nodes (triangles) that lie in the star of a vertex of core $(\Gamma)$.

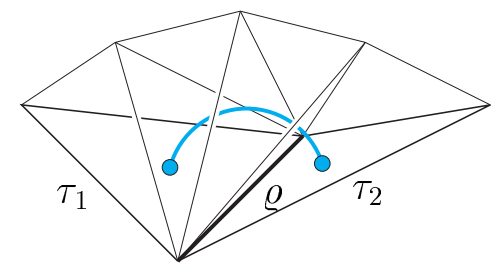

Fig. 12

Lemma 11. Let $\varrho$ be a 1-face of the triangle $\tau \in \operatorname{core}(\Gamma)$. Then there is at least one more triangle $\tau^{\prime} \in \operatorname{core}(\Gamma)$ which contains $\varrho$, and is joined to $\tau$ by an edge in $G_{\mathcal{B}}^{*}$.

Proof. $\tau$ is covered from both sides with tetrahedra $\sigma_{\uparrow}$ and $\sigma_{\downarrow} \in \operatorname{core}(\Gamma)$. The vertices $v\left(\sigma_{\uparrow}\right) \neq v\left(\sigma_{\downarrow}\right)$ are both vertices of $\tau$, so that every edge of $\tau$ contains at least one of them.

Corollary 12. For every vertex $v \in \operatorname{core}(\Gamma)$, the subgraph of $G_{\mathcal{B}}^{*}$ induced by the triangles incident to $v$ is 2-connected.

Proof. If $v$ is a regular vertex (all incident edges have degree 2 in core $(\Gamma)$ ), then the corresponding local subgraph of $G_{\mathcal{B}}^{*}$ is a cycle: it is the dual graph of the star of $v$ in $\operatorname{core}(\Gamma)$.

If $v$ is a singular vertex (two incident edges have degree 3 in core $(\Gamma)$ ), then the subgraph of $G_{\mathcal{B}}^{*}$ is a union of three paths between two nodes. 


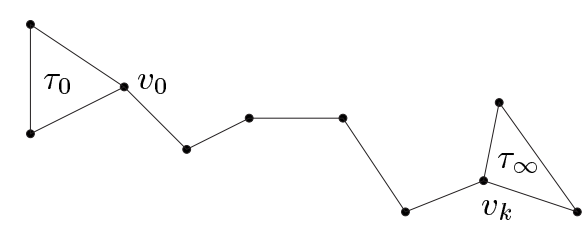

Fig. 13

Now, to verify 3-connectivity of $G_{\mathcal{B}}^{*}$, we use the following simple but very useful observation of Naatz:

Theorem 13 [18, Theorem 3.3]. A graph $G$ on at least $k+1$ vertices is $k$-connected if and only if for every two vertices $v$ and $w$ at distance 2 there are at least $k$ independent $v$-w paths in $G$.

Proposition 14. $G_{\mathcal{B}}^{*}$ is 3-connected.

Proof. Remove two nodes/triangles $\bar{\tau}_{1}, \bar{\tau}_{2}$ from $G_{\mathcal{B}}^{*}$, and denote $\tau_{0}, \tau_{\infty}$ the two nodes which we want to join by a $\left(\bar{\tau}_{1}, \bar{\tau}_{2}\right)$-avoiding path. In core $(\Gamma)$, the intersection $\bar{\tau}_{1} \cap \bar{\tau}_{2}$ contains at most two vertices. Now the 1-skeleton of core $(\Gamma)$ is 3 -connected (e.g. by Naatz' lemma). Thus there is a $\left(\bar{\tau}_{1} \cap \bar{\tau}_{2}\right)$-avoiding vertex-edge path in core $(\Gamma)$ from a vertex of $\tau_{0}$ to some vertex of $\tau_{\infty}$. That is, there are vertices $v_{0}, \ldots, v_{k}$ of core $(\Gamma)$ such that $v_{0} \in \tau_{0}, v_{k} \in \tau_{\infty}$, and the $\left\{v_{i}, v_{i-1}\right\}$ 's are edges of core $(\Gamma)$ (see Fig. 13). Choose triangles $\tau_{i} \in \operatorname{core}(\Gamma)$ adjacent to the edges $\left\{v_{i-1}, v_{i}\right\}$. By Corollary 12, consecutive triangles $\tau_{i}$ and $\tau_{i+1}$ can be joined within the star of $v_{i}$, avoiding $\bar{\tau}_{1}, \bar{\tau}_{2}$ (see Fig. 14).

Finally, we get Step 3 for free:

Corollary 15. $\Gamma$ is dually 3-connected.

Proof. Because core $(\Gamma)$ is induced, $\bar{\sigma}_{i} \cap \operatorname{core}(\Gamma)$ is a face of $\bar{\sigma}_{i}$. If $\bar{\sigma}_{i} \cap \operatorname{core}(\Gamma)$ is a triangle, remove the corresponding node from $G_{\mathcal{B}}^{*}$. If $\bar{\sigma}_{i} \cap \operatorname{core}(\Gamma)$ is an edge, remove the edge from $G_{\mathcal{B}}^{*}$, which is cut by $\bar{\sigma}_{i}$. By Lemma 10 , we can assume that we want to join two tetrahedra $\sigma_{0}, \sigma_{\infty} \in \Gamma$ which intersect core $(\Gamma)$ in triangles $\tau_{1}$ and $\tau_{2}$, respectively. By Proposition 14 we can find a path from $\tau_{1}$ to $\tau_{2}$ in the remaining graph. This path can be lifted to a dual path which joins $\sigma_{0}$ and $\sigma_{\infty}$, and which avoids $\bar{\sigma}_{1}, \bar{\sigma}_{2}$.

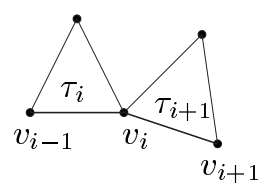

Fig. 14 


\section{Remarks}

It seems reasonable to ask whether every induced, 3-connected, 3-regular, non-separating and planar (!) subgraph of the graph of a simple 4-polytope is the graph of a facet. For $d>4$, the natural condition that could replace the "planar" would be "isomorphic to the graph of a $(d-1)$-polytope." However, there is no effective (polynomial time) method available to check whether this condition is satisfied, for $d>4$.

Does the 120-cell satisfy the Perles conjecture?

\section{Acknowledgments}

Thanks, in particular, to Tom Braden, Wlodek Kuperberg, Carsten Lange, Mark de Longueville, Carsten Schultz, Raik Stolletz, Niko Witte and Elmar Vogt for crucial discussions.

\section{References}

1. H. Achatz and P. Kleinschmidt: Reconstructing a simple polytope from its graph, in: PolytopesCombinatorics and Computation (G. Kalai and G. M. Ziegler, eds.), DMV Seminar, Vol. 29, Birkhäuser, Basel, 2000, pp. 155-165.

2. R H Bing: Some aspects of the topology of 3-manifolds related to the Poincaré conjecture, in: Lectures on Modern Mathematics, Vol. II (T. L. Saaty, ed.), Wiley, New York, 1964, pp. 93-128.

3. A. Björner: Shellable and Cohen-Macaulay partially ordered sets, Trans. Amer. Math. Soc. 260 (1980), 159-183.

4. R. Blind and P. Mani-Levitska: On puzzles and polytope isomorphisms, Aequationes Math. 34 (1987), 287-297.

5. G. Bohus, W. Jockusch, C. W. Lee, and N. Prabhu: On a triangulation of the 3-ball and the solid torus, Discrete Math. 187 (1998), 259-264.

6. K. Borsuk: Über das Phänomen der Unzerlegbarkeit in der Polyedertopologie, Comment. Math. Helv. 8 (1935), 142-148.

7. E. Gawrilow and M. Joswig: polymake: a framework for analyzing convex polytopes, in: PolytopesCombinatorics and Computation (G. Kalai and G. M. Ziegler, eds.), DMV Seminar, Vol. 29, Birkhäuser, Basel, 2000, pp. 43-73.

8. E. Gawrilow and M. Joswig: Polymake: a framework for analyzing convex polytopes, 1997-2000, http://www.math.tu-berlin.de/diskregeom/polymake/doc/.

9. B. Grünbaum: Convex Polytopes, Interscience, London, 1967.

10. A. Hatcher: Algebraic Topology, www.math.cornell.edu/ $\sim$ hatcher/, Cornell University, 2000; Cambridge University Press, 2002.

11. F. Holt and V. Klee: Counterexamples to the strong $d$-step conjecture for $d \geq 5$, Discrete Comput. Geom. 19 (1998), 33-46.

12. M. Joswig: Reconstructing a non-simple polytope from its graph, in: Polytopes-Combinatorics and Computation (G. Kalai and G. M. Ziegler, eds.), DMV Seminar, Vol. 29, Birkhäuser, Basel, 2000, pp. 167176.

13. M. Joswig, V. Kaibel and F. Körner: On the $k$-systems of a simple polytope, Israel J. Math. 129 (2002), 109-118.

14. G. Kalai: A simple way to tell a simple polytope from its graph, J. Combin. Theory Ser. A 49 (1988), 381-383.

15. G. Kalai: Some aspects of the combinatorial theory of convex polytopes, in: Polytopes: Abstract, Convex and Computational (T. Bisztriczky, P. McMullen and A. Weiss, eds.), Proc. NATO Advanced Study Institute, Toronto, 1993, Kluwer, Dordrecht, 1994, pp. 205-230. 
16. V. Klee and D. W. Walkup: The $d$-step conjecture for polyhedra of dimension $d<6$, Acta Math. 117 (1967), 53-78.

17. J. R. Munkres: Elements of Algebraic Topology, Addison-Wesley, Reading, MA, 1984.

18. M. Naatz: The graph of linear extensions revisited, SIAM J. Discrete Math. 13 (2000), 354-369.

19. M. Perles: Results and problems on reconstruction of polytopes, Jerusalem, 1970, unpublished [14]; problems posed in Oberwolfach 1984 and 1986 [4].

20. J. Pfeifle and G. M. Ziegler: Cyclic polytopes and their duals, in preparation.

21. R. Stolletz: Facetten-Graphen einfacher Polytope, Diplomarbeit, TU Berlin, 1998, 77 pages.

22. H. Whitney: Non-separable and planar graphs, Trans. Amer. Math. Soc. 34 (1932), 339-362.

23. N. Witte: A counterexample to the Perles conjecture, Electronic geometry model, www.eg-models.de, in preparation.

24. E. C. Zeeman: On the dunce hat, Topology 2 (1963), 341-358.

25. G. M. Ziegler: Lectures on Polytopes, Graduate Texts in Mathematics, Vol. 152, Springer-Verlag, New York, 1995; revised edition, 1998.

Received July 19, 2001, and in revised form December 6, 2001. Online publication April 19, 2002. 